\title{
Mays Cancer Center
}

National Cancer Institute

\section{Source}

National Cancer Institute. Mays Cancer Center. NCI Thesaurus. Code C157460.

A cancer center established by UT Health San Antonio in the 1970s that achieved NCIDesignated Cancer Center status in 1991 and is affiliated with MD Anderson Cancer Center. 